\title{
La planificación y competitividad turística ecuatoriana en el contexto del geoparque Imbabura.
}

\section{Ecuadorian tourism planning and competitiveness in the context of the geopark Imbabura.}

MSc. Yoarnelys Vasallo Villalonga. ${ }^{1}$, Dra. Bertha Soraya Rhea González. ${ }^{2}$ \& Dra. Adelfa María La Serna Gómez. ${ }^{3}$

\section{Resumen.}

Ecuador es un país con amplia variedad de atractivos naturales y manifestaciones culturales. La presente investigación se enfocó a analizar la planificación y competitividad turística en el país, en el contexto del Geoarque Imbabura, declarado de interés prioritario por la Comisión Nacional Ecuatoriana de la UNESCO. Se indagó sobre los planes y programas de desarrollo turístico, direccionados desde la empresa pública e iniciativas de emprendimientos privados, y sus condiciones de calidad en la prestación de servicios turísticos. Se utilizaron métodos de investigación como el analítico-sintético y el histórico-lógico y técnicas como: observación directa, entrevistas y encuestas. Los resultados obtenidos sustentan una metodología de evaluación -de la calidad- a través de parámetros establecidos por el MINTUR y por proyectos comunitarios con características afines al área de estudio, a partir del cumplimiento de la normativa legal. Se realizó un estudio de mercado para conocer el perfil del cliente.

Palabras claves: Geoparque, planificación, competitividad, calidad, UNESCO.

\section{Abstract.}

Ecuador is a country with a wide variety of natural attractions and cultural manifestations. This research focused on analyzing tourism planning and competitiveness in the country, in the context of the Imbabura Geopark, declared of priority interest by the UNESCO

\footnotetext{
${ }^{1}$ Universidad Técnica de Norte. Ibarra. Ecuador. yvasallo@utn.edu.ec

${ }^{2}$ Universidad Técnica de Norte. Ibarra. Ecuador. srheag@utn.edu.ec

${ }^{3}$ Universidad Técnica de Norte. Ibarra. Ecuador. amlaserna@utn.edu.ec
} 
Ecuadorian National Commission. It inquired about the plans and programs of tourist development, directed from the public company and initiatives of private enterprises, and their conditions of quality in the provision of tourist services. We used research methods such as analytical-synthetic and historical-logical techniques such as direct observation, interviews and surveys. The results obtained propose an evaluation methodology -of quality- through parameters established by MINTUR and by community projects with characteristics related to the study area, based on compliance with legal regulations. A market study was conducted to know the customer's profile.

Keywords: Geopark, planning, competitiveness, quality, UNESCO.

\section{Introducción.}

Los Geoparques representan una estrategia de desarrollo territorial sostenible impulsada y reconocida por la UNESCO mediante el Programa Internacional de Geociencia y Geoparques. Entre las tareas principales consta la conservación de los patrimonios, la educación, el desarrollo y el bienestar de las comunidades, teniendo como base el aprovechamiento del geoturismo.

El geoparque Imbabura fue declarado de interés prioritario por la Comisión Nacional Ecuatoriana de la UNESCO desde diciembre del 2016. Para que un territorio sea reconocido como Geoparque Mundial UNESCO debe contener un patrimonio geológico claramente identificado y caracterizado que se utilice de forma sostenible para el desarrollo económico local (UNESCO, 2016). En este sentido la UNESCO alienta al trabajo con instituciones académicas para participar en la investigación científica activa que propicie el desarrollo sostenible, el conocimiento local e indígena y la geoconservación, entre otros [1].

En los marcos de la investigación no se han identificado estudios precedentes que incidan en el desarrollo del geoparque, motivo por el cual se aboga por el establecimiento de vínculos investigativos que permitan a la universidad incidir en la solución de los problemas de la sociedad imbabureña y a su vez, crear las bases para impulsar el reconocimiento internacional de la puesta en valor de los atractivos del parque.

Ante esta situación el problema de investigación que se plantea es cómo contribuir al desarrollo sustentable e inclusivo del Geoparque Imbabura a través de la planificación y la competitividad turística, con evaluación de las condiciones de calidad en la prestación de servicios.

El Gobierno Provincial de Imbabura (GPI) en julio del 2015, resolvió apoyar la implementación del Proyecto Geoparque Imbabura, en el cual están representados los 6 cantones y 36 parroquias rurales que ofrecen a sus visitantes algunas modalidades de turismo; mismas que se han ido desarrollando en el transcurso del tiempo.

La amplia extensión geográfica, la inclusión de una población rural significativa con problemas pendientes a solución, la diversidad étnica y la riqueza de atractivos naturales y culturales no valorizados, son algunos de los argumentos que justifican la importancia y pertinencia de la investigación, direccionada a contribuir al posicionamiento de 
Imbabura y Ecuador como destinos turísticos de nivel internacional, con reconocimiento de la UNESCO, mediante la declaratoria de Geoparque Internacional.

En este contexto, la investigación que se presenta plantea como objetivos: analizar la planificación y competitividad turística en el país, en el contexto del Geoarque Imbabura, a través de la indagación sobre los planes y programas de desarrollo turístico, direccionados desde el gobierno nacional y la empresa pública e iniciativas de emprendimientos privados, y sus condiciones de calidad en la prestación de servicios turísticos y realizar el estudio de mercado para conocer el perfil del cliente que los adquiere.

Los estándares de calidad turística son de alta importancia y determinan fuertemente la llegada de los turistas a un destino, dejando de lado a aquellos que no satisfacen las expectativas creadas, en muchas ocasiones, por estrategias de publicidad engañosa. Cabe recalcar que la calidad no necesariamente supone altos costos, sino más bien, al cumplimiento de requisitos mínimos establecidos a cualquier escala de gasto, que permitan generar satisfacción y complacencia en los clientes, respetando además criterios de protección del patrimonio ambiental y cultural de las zonas vinculadas a la recepción turística.

Ofrecer servicios de calidad, estimular las inversiones turísticas y fortalecer la promoción interna y externa del potencial turístico de Ecuador, fueron los principales ejes de acción del Ministerio de Turismo de Ecuador durante 2015; año que fue declarado el Año de la Calidad Turística; pues uno de cada 20 empleos se encuentra en este sector terciario. Es así como 278 empresas de la ciudad de Quito cuentan con la marca Q otorgada por el Sistema Nacional de Calidad Turística del MINTUR.

En este mismo sentido las campañas que ha promovido el gobierno para dar a conocer los atractivos naturales y manifestaciones culturales como: Ecuador Ama la vida, Ecuador Potencia turística, All you need is Ecuador; tienen además el objetivo de motivar a los prestadores de servicios a la mejora continua y a lograr la competitividad frente a otros destinos. Y es que las realidades turísticas se han modificado mucho en los últimos años, tanto o más que las perspectivas futuras. Como indicaran Vega y Vasallo, (2017), por muchos años el turismo se reservó como actividad de lujo solo para las grandes élites burguesas, sin embargo con posterioridad a la segunda guerra mundial la evolución de determinados factores económicos, políticos, sociales y tecnológicos le generalizaron como un fenómeno social de alcance para las burguesías medias, entre ellas la traspolación a la aviación comercial de los avances de la aviación militar, el desarrollo de otras formas de transporte y de los medios de comunicación masiva, el derecho al descanso retribuido y el aumento del nivel cultural [2].

En la actualidad, el turismo es una actividad en ascenso que se fortalece y adquiere cada vez, mayor relevancia y participación en el desarrollo económico de los territorios que participan del movimiento turístico internacional. En Ecuador, el desarrollo y gestión de la actividad turística se ha hecho presente en la agenda nacional como política de gobierno. Como para muchos otros países, este sector ha sido en los últimos años, un eje fundamental en la reactivación económica, con indicadores importantes como la generación de empleos, los proyectos de inversión local y extranjera, el desarrollo de 
infraestructura hotelera y vías de acceso, las conexiones aéreas con vuelos domésticos e internacionales, etc, que han favorecido la atracción de divisas a la economía nacional.

\section{Métodos}

En este sentido, se trata de una investigación aplicada, con utilización de métodos cuantitativos y cualitativos para el análisis de la información. Para analizar la planificación y competitividad turística en el país, se realizó investigación bibliográfica con la revisión de documentos y aplicación de métodos teóricos de la investigación como analítico-sintético e histórico-lógico, lo cual permitió indagar sobre los planes y programas de desarrollo turístico generados en el país desde el gobierno nacional y la empresa pública. Para determinar las condiciones de calidad en la prestación de servicios turísticos en las iniciativas de emprendimientos privados, de aplicó una ficha de observación que sustenta la metodología propuesta para la evaluación de las condiciones de calidad en la prestación de servicios turísticos, previa revisión documental de la normativa legal vigente desde el MINTUR y sus reglamentos y legislaciones. Y para la realización del estudio de mercado se aplicaron guías de observación directa y un cuestionario con preguntas, en su mayoría cerradas, que permitieron identificar el perfil del cliente y su nivel de satisfacción.

\section{Resultados}

Gran parte del patrimonio ecuatoriano se encuentra en las parroquias rurales. En el caso de las de Imbabura, mayormente viven en entornos interculturales de familias indígenas y mestizas, hablan el castellano y el quechua y conservan sus costumbres, tradiciones y leyendas. Se dedican mayoritariamente a actividades de sobrevivencia como la agricultura y la crianza de animales domésticos. Algunas elaboran bordados a mano en prendas de vestir con un acabado y gusto apreciables que han traspasado las fronteras nacionales. Otras familias se dedican a los talleres de talabartería que confeccionan monturas, maletas y artefactos finos. El desarrollo de la actividad turística les ha permitido organizarse para ofertar servicios turísticos, esencialmente alojamiento y alimentación.

Si se analiza la calidad en su gestión, los resultados a priori son discretos. Se percibe desconocimiento de cuestiones fundamentales con alto empirismo en la prestación de servicios y el consecuente desaprovechamiento de gran parte del potencial natural y cultural que poseen. Siendo así, es necesario articular un trabajo multidisciplinario con profesionales técnicos para tomar en cuenta a otros lugares que también realizan actividades turísticas, con la intención de complementarse y presentar paquetes más completos y atractivos que permitan incrementar la oferta y demanda turística.

En estas parroquias rurales existen mayormente establecimientos de alojamiento turístico y restauración, registrados todos en los Catastros de Emprendimientos Turísticos, los cuales son en su mayoría de carácter comunitario: casas de hospedaje, refugios y hosterías, según la clasificación que rige a nivel nacional. 
Para identificar los elementos condicionantes de la calidad en estos establecimientos, se toma como punto de partida la normativa vigente, la cual considera parámetros, indicadores, lineamientos y artículos que permiten regular los servicios turísticos.

El Ministerio de Turismo como ente rector de la actividad turística a nivel nacional, deriva toda la normativa de la Ley de Turismo, de la cual se deprenden los reglamentos de actividades turísticas actuales y en vigencia, organizadas en alojamiento turístico, intermediación y operación turística y el de guianza.

En los reglamentos de turismo comunitario y alimentos y bebidas se está trabajando recién, para que sean concebidos con registro oficial y consecuentemente puedan establecerse normas y estándares nacionales para estos tipos de actividades. A la fecha, solo la Ley de Turismo y el Reglamento de Alojamiento Turístico son los instrumentos vigentes para la evaluación de calidad [3;4].

La planta central del MINTUR y cada una de las coordinaciones zonales, dispone de la Unidad de Registro y Control, cuya función explícita consiste en verificar y controlar el buen funcionamiento y la prestación de servicios en los establecimientos que han sido categorizados como turísticos, a través de operativos de control, en los que se aplican check list de verificación del cumplimiento de indicadores.

Con la estrategia de zonificación se han identificado en una hoja de ruta, todos los establecimientos reconocidos en el catastro que brindan servicios turísticos, independientemente de su ubicación geográfica. Los controles los realiza el MINTUR a través de auditorías. En los casos que lo requieren, se realizan adicionalmente, asesoramiento a los propietarios, y existe además, una plataforma de capacitación on-line, con programas específicos de las diferentes temáticas, como manipulación de alimentos, atención al cliente, camareras y pisos, recepción y gerencia.

Para los alojamientos turísticos los aspectos tomados en cuenta para la evaluación son: infraestructura en buen estado, área de recepción, sistema de quejas y sugerencias, mobiliario de habitaciones, limpieza de áreas exteriores, seguridad, hospitalidad del personal, baños y grifos funcionales, áreas de cocina y comedor en estado funcional, manipulación y preparación de alimentos, bodegas y áreas de almacenamiento de alimentos, áreas de descanso, sostenibilidad ambiental con prácticas correctas del manejo de desechos, desarrollo en general del establecimiento y áreas de emergencia.

La ficha de observación utilizada otorga una calificación cuantitativa a las prestaciones de cada una de las áreas de los establecimientos de alojamiento. En base a este criterio la calificación máxima es 475 puntos, teniendo en cuenta que los aspectos a calificarse se han establecido en un rango de 5 puntos (Excelente), 4 puntos (Muy Bueno), 3 puntos (Bueno), 2 puntos (Regular) y 1 punto (Malo).

Los requerimientos calificados son: aspectos generales, operación y servicio, habitaciones, baños para huéspedes, cocina y área de comedor, alimentación y bebidas, áreas de descanso, seguridad y aspectos de sostenibilidad ambiental. Cada uno de ellos posee subdivisiones en los cuales hay ítems que se ha considerado deben ser aplicables en la prestación del servicio de alojamiento para lograr la satisfacción de los clientes. 
Para identificar el perfil del cliente que consume los establecimientos turísticos rurales del Geoparque Imbabura, se han elaborado cuestionarios con preguntas abiertas y cerradas. Para la cobertura de las unidades de análisis se aplica la fórmula de cálculo de la muestra y en el procedimiento para tratamiento y análisis de la información obtenida, se utiliza la estadística descriptiva.

Se toma como población el número de turistas que ingresan a la parroquia La Esperanza, una de las más representativas de la provincia. Se aplica un censo al 100\% de la población, conformada por 150 clientes, según datos empíricos ofrecidos por los propietarios, al carecer de un registro de ingreso de clientes. Para el servicio de alimentos y bebidas se ha identificado la existencia de cinco locales, en los que prevalece una demanda estacional de fines de semana, que promedia los 800 clientes. Cada muestra respondió su propio cuestionario.

Los resultados de la encuesta dirigida a los clientes que adquieren el servicio de alojamiento, indican que la edad se encuentra mayormente entre los 26 y 50 años, con una ligera tendencia hacia el género masculino en un 52,7\%. El nivel de ingresos oscila entre $\$ 501$ y $\$ 1200$ mensuales para el 67,4\%. Provienen de Quito en un 49,3\% y de Estados Unidos, Francia y Alemania en 18\%, 14\% y $8 \%$ respectivamente. Como promedio pernoctan entre una y dos noches, y regresan una o dos veces al año. Para elegir dónde hospedarse, el $42,7 \%$ acudió a redes sociales y un $38 \%$ a páginas de internet.

Por su parte los clientes que respondieron la encuesta de consumo de servicios de alimentos y bebidas en un 73,8\% se encuentran en edades entre los 26 y 50 años, el 65,4\% pertenece al género femenino, que generalmente viaja en familia los fines de semana. En la procedencia, se destaca Ibarra con el 53.1\%, en mucha menor medida Quito, Atuntaqui y Otavalo con el 28,5\%, 4,2\% y 3,8\% respectivamente. El 9,2\% de los clientes son personas de la localidad y barrios cercanos de la parroquia.

A la frecuencia de consumo, el 54,2\% indica 2 veces al mes, el 27,3\%, una y el 11,2\%, 3 veces al mes, lo cual se corresponde con la cercanía de la parroquia a las ciudades emisoras, la calidad de la cocción y los precios asequibles de los platos, que en un 76,1\% oscila entre $\$ 3.01$ y $\$ 5,00$.

\section{Discusión}

No podría afirmarse aún, que el turismo sea como se aspira, el nuevo petróleo ecuatoriano, pero los esfuerzos de las iniciativas implementadas ya comienzan a percibirse. A la fecha, es el tercer sector productivo no petrolero que más divisas genera, las cuales contribuyen a reducir inequidades y desigualdades, mejorar la calidad de vida, generar empleos, descanso, recreación y nuevas oportunidades; y no solo para el turismo internacional, sino que concibe el acceso al viaje y al descanso para la mayoría de los ecuatorianos, con el decreto de ajustes a los feriados nacionales a fin de prolongar los fines de semana, la construcción de vías de acceso de primer orden, la presencia de paradores turísticos en ubicaciones estratégicas, etc. Se procura un turismo consiente, una actividad ética y sostenible que pueda producir una experiencia transformadora de vida, a la vez que genera 
nuevas oportunidades para pequeños y medianos emprendimientos. (Vasallo y Arciniegas, 2015) [5].

La variedad de paisajes, culturas, grupos étnicos, flora y fauna, así como las numerosas posibilidades de realizar turismo vivencial, ya sea de sol y playa, científico, rural y de montaña, de aventura, urbano o de ciudad, gastronómico, de negocios, entre otros, lo convierten en un destino atractivo.

Consecuentemente, desde el gobierno nacional se han estructurado en algunos casos, y actualizado en otros, una serie de programas y proyectos que rigen la planificación y política turística del país, con visiones definidas a corto, mediano y largo plazo, entre ellos el Plan Estratégico de Desarrollo de Turismo Sostenible, PLANDETUR 2020 (2007), Plan Integral de Marketing Turístico 2014 PIMTE (2014), el Programa Nacional de Destinos Turísticos de Excelencia (2014) y el Programa Nacional para la Excelencia Turística (2015). (Vasallo y Vega, 2018) [6].

El PLANDETUR 2020, (2007) busca consolidar al turismo sostenible como uno de los ejes dinamizadores de la economía ecuatoriana, a partir del aprovechamiento de sus ventajas competitivas y de la inversión en facilidades turísticas, generando una adecuada gestión de los territorios y líneas de productos [7].

Redactado a través de proceso participativo por la empresa Tourism \& Leisure Advisory Services contratada por el Ministerio de Turismo, y financiada por el Banco Interamericano de Desarrollo (BID), se direcciona a potencializar las condiciones de la oferta nacional y fomentar el desarrollo local que genere oportunidades equitativas en el marco del Buen Vivir.

Por su parte el PIMTE 2014, (2014) se orienta a posicionar al Ecuador como un destino turístico de referencia a nivel mundial con el objetivo de incrementar los arribos internacionales y los desplazamientos internos, que generen el ingreso de divisas a la economía nacional en el primer caso, y en el segundo se promueva la generación de empleos, la equidad en la distribución de los ingresos, la orientación a la calidad en el servicio al turista y la dinamización general de la cadena productiva del turismo, con el establecimiento de un sistema económico social, solidario y sostenible [8].

En el caso del Programa Nacional de Destinos Turísticos de Excelencia (2015), el objetivo principal es potenciar competitivamente mediante cuatro componentes, la oferta turística del Ecuador a través de la consolidación y desarrollo de rutas, circuitos, destinos y atractivos turísticos priorizados a fin de contribuir con la excelencia para el sector turístico [9].

Por su parte, el Programa Nacional para la Excelencia Turística (2015), se plantea para mejorar la calidad de la oferta de servicios turísticos, mediante la implementación de acciones y proyectos concretos como el desarrollo de normativas y protocolos de calidad; la implementación de certificaciones e incentivos; la formación y capacitación del talento humano; la investigación de nuevos modelos y tendencias en la gestión y el desarrollo turístico y la gestión y sensibilización de la población hacia una cultura de innovación y excelencia. (MINTUR, 2015) [10]. 
Las características naturales y socio-demográficas de Ecuador, le convierten en un país con posibilidades reales de desarrollo y crecimiento turístico. La interacción de una geografía privilegiada, gran acervo cultural, monumentos arqueológicos e históricos, son solo algunos atractivos, de los muchos que generan interés, como sería el caso del Geoparque Imbabura, que desde los andes ecuatorianos ofertaría a sus visitantes algunas modalidades de turismo.

En este sentido, las Naciones Unidas, en el marco de los Objetivos de Desarrollo Sostenible (2015-2030), reconocen en el Objetivo 8 la necesidad de promover el crecimiento económico sostenido, inclusivo y sostenible, el empleo pleno y productivo y el trabajo decente para todos [11].

Y la Organización Mundial del Turismo, (OMT), en su Código Ético Mundial para el Turismo, (OMT, 1999), que si bien es cierto, no es un documento jurídicamente vinculante, sí es una referencia necesaria a tomar en cuenta e implementar por parte de los actores de la actividad turística, en sus Artículos 2 y 3, había referido al turismo como instrumento de desarrollo profesional y colectivo y como factor de desarrollo sostenible [12].

En Ecuador, las políticas públicas y los planes de gobierno para el crecimiento del sector turístico destacan entre sus fortalezas la intención manifiesta de no dar continuidad a los modelos tradicionales de consumismos en los que imperan los indicadores de beneficio económico por sobre los socioculturales y ambientales, sino que se potencian modalidades como el turismo cultural, patrimonial y ecológico, incrementándose las visitas a áreas protegidas y a ecosistemas frágiles, como la iniciativa del GPI de incentivar el desarrollo sustentable e inclusivo del Geoparque Imbabura.

Al modificar la forma de cómo se ha desarrollado la economía en el Ecuador se busca una alternativa de desarrollo que no afecte los recursos naturales ni los comprometa a futuro. De esta manera el turismo desempeña una función importante en la consecución de estos objetivos, al convertirse en una posible alternativa de desarrollo con potencialidades reales, para el presente y el futuro de la economía nacional.

Las campañas que ha promovido el gobierno para dar a conocer los atractivos naturales y manifestaciones culturales como: Ecuador Ama la vida, Ecuador Potencia turística, All you need is Ecuador, tienen además el objetivo de motivar a los prestadores de servicios en la mejora continua y potenciar con ello la competitividad frente a otros destinos.

Según las estadísticas manejadas por el Gobierno Provincial de Imbabura, el turismo en la provincia ha crecido un 9,3\% en el año 2017 con respecto al año anterior, y los establecimientos turísticos han crecido en un 4,3\%. Con ello, Imbabura se encuentra entre las cinco provincias receptoras de turismo en el país. El Cantón Ibarra y sus parroquias rurales ofrecen a sus visitantes algunas modalidades de turismo, mismas que se han ido desarrollando en el transcurso del tiempo.

La nueva iniciativa del Geoparque Imbabura, desde su concepción de desarrollo sustentable e inclusivo, contribuirá al posicionamiento de Imbabura y Ecuador como destinos turísticos de nivel internacional. 


\section{Conclusiones.}

$>$ Se analizaron los planes y programas de desarrollo turístico que direccionados desde la empresa pública tributan a la planificación y competitividad turística del país.

$>$ Se sustentó una metodología de evaluación de la calidad, que a través de parámetros establecidos por el MINTUR y por proyectos comunitarios con características afines al área de estudio, evalúa la calidad en los servicios turísticos, a partir del cumplimiento de la normativa legal vigente.

$>$ Se realizó un estudio de mercado que permitió identificar el perfil de los clientes que adquieren servicios turísticos rurales en la provincia, como aproximación a la demanda potencial del Geoparque Imbabura..

\section{Referencias Bibliográficas.}

[1]. URUGUAY. Organización de las Naciones Unidad para Educción, la Ciencia y la Cultura. Programa Internacional de Geociencias y Geoparques. Montevideo, Uruguay 2016. Disponible en: http://www.unesco.org/new/es/office-inmontevideo/natural-sciences/international-programme-for-geosciences-andgeoparks/

[2]. VEGA FALCÓN, V. y VASALlO VILlALONGA, Y. Planificación y Competitividad Turística: Caso Ecuador. Quito, Ecuador: Ed. El Siglo, 2017. 204 p. ISBN: 978-9942-30-500-8.

[3]. ECUADOR. Ministerio de Turismo. Ley Orgánica de Turismo. Registro Oficial Suplemento 733 de 27-dic-2002, última modificación: 29-dic-2014. Quito, Ecuador: 2014.

[4]. ECUADOR. Ministerio de Turismo. Reglamento de Alojamiento Turístico. RO.465. Quito, Ecuador 2015.

[5]. VASALlO VILLALONGA, Yoarnelys y ARCINIEGAS PASUEL, Edison. Desarrollo sostenible de la actividad turística: realidad y perspectivas. Revista Ecos de la Academia. 1 (1): 147 - 163, 2015. ISSN: 1390-969X

[6]. VASALlO VILlalONGA, Yoarnelys y VEGA FALCÓN, Vladimir. La planificación y competitividad turística en Ecuador. Revista Metanoia. 3 (5), 4960, 2018. ISSN: 1390-9282.

[7]. ECUADOR. Ministerio de Turismo. Plan Estratégico de Desarrollo de Turismo Sostenible para Ecuador, PLANDETUR 2020. Quito, Ecuador 2007. Disponible en: http://www.turismo.gob.ec/wp content/uploads/downloads/2013/02/PLANDETUR-2020.pdf

[8]. ECUADOR. Ministerio de Turismo. Plan Integral de Marketing Turístico de Ecuador PIMTE 2014. Quito, Ecuador 2014. Disponible en 
http://www.turismo.gob.ec/wpcontent/uploads/downloads/2013/02/PIMTE_201 4.pdf

[9]. ECUADOR. Ministerio de Turismo. Programa Nacional de Destinos Turísticos de Excelencia. Quito, Ecuador 2015. Disponible en: http://www.turismo.gob.ec/wpcontent/uploads/downloads/2015/09/Literal-k-Planes-y-programas-Agosto2015.pdf

[10] ECUADOR. Ministerio de Turismo. Programa Nacional para la Excelencia Turística. Quito, Ecuador 2015. Disponible en: http://www.turismo.gob.ec/wpcontent/uploads/2015/04/Documento-Programa-Nacional-para-la-ExcelenciaTuristica1.pdf

[11]. Organización Mundial del Turismo. Objetivos de Desarrollo Sostenible 2015-2030. El sector turístico y los objetivos de desarrollo sostenible. Turismo responsable, un compromiso de todos. 2016. Disponible en: http://www.thinktur.org/media/Publicacin-Turismo-Responsable-uncompromiso-de-todos.pdf

[12]. Organización Mundial del Turismo. Código Ético mundial para el Turismo. Santiago de Chile, Chile, 1999.

\section{\Ciencia}




\section{Para citar el artículo indexado.}

Vasallo Y., Rhea B. \& La Serna A. (2019) La planificación y competitividad turística ecuatoriana en el contexto del geoparque Imbabura. Revista electrónica Explorador Digital 3(3), 15-25. Recuperado desde:

http://cienciadigital.org/revistacienciadigital2/index.php/exploradordigital/article/view/440/9 $\underline{88}$

\section{Ciencia \\ Digital \\ Editorial}

El artículo que se publica es de exclusiva responsabilidad de los autores y no necesariamente reflejan el pensamiento de la Revista Explorador Digital.

El articulo queda en propiedad de la revista y, por tanto, su publicación parcial y/o total en otro medio tiene que ser autorizado por el director o editor de la Revista Explorador Digital.
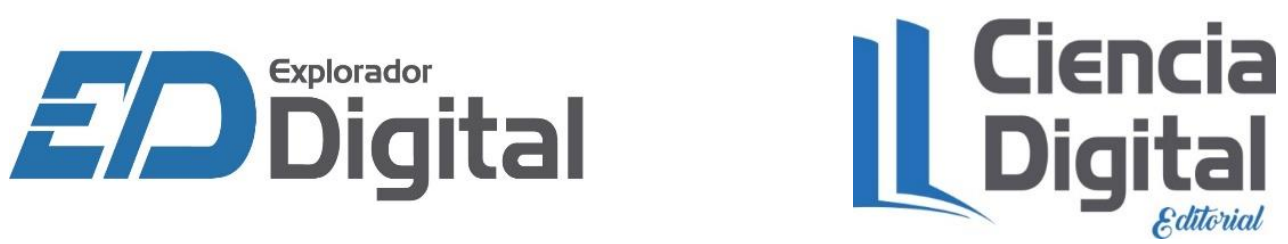